\title{
The Development of an AGVS Model by the Union of the Modulised Floor-Path Nets
}

\author{
Suhua Hsieh and Ying-Jer Shih \\ Department of Mechanical Engineering, National Taiwan University, Taipei, ROC
}

The purpose of this paper is to propose an easy and quick method for the development of an automated guided vehicle system (AGVS) model. It suggests that the AGVS model can be developed directly by the union of modulised floor-path nets without any modification. Several modulised floor-path nets for both uni-and bi-directional systems are established in this paper. To make sure that the complete model obtained by this method is robust, the modulised floor-path nets are required to possess several essential properties - safeness, boundedness, conservation, reachability and liveness. To keep these properties in the complete model after the union, three union rules and an union procedure are proposed.

Keywords: AGVS; Petri-net properties; Robust; Union

\section{Introduction}

As mentioned in $[1,2]$, to build a robust automated guided vehicle system (AGVS) needs very carefully study and design from different aspects (such as the existing plant layout, the manufacturing processes, the cooperate management policies, etc.). In [3], a three-level (vehicle-level, floor-level and management-level) robustness concept for an AGVS has been specified. The technology of the vehicle level is relatively mature when compared to that of the other two levels. This is because most new users usually do not realise that the AGVS design has three levels and the design at the second and third levels depends on the type of application. Therefore, users are often not involved in the design of the AGVS as much as they should be. This results in the failure of the AGVS. The design at the management level is totally casedependent. Fortunately, the design at shop floor level becomes common to every case, if the floor-path layout is determined.

Correspondence and offprint requests to: Sushua Hsieh, Department of Mechanical Engineering, National Taiwan University, 1 Roosevelt Road Sec 4, Taipei, Taiwan 10764, Republic of China.
The purpose of this paper is to propose an easy and quick modelling tool which can help users to build a robust system at shop floor level. The modelling method is to establish several basic modulised floor-path nets, and then, by the union of these nets, a complete system model can easily be developed.

A similar idea has been proposed in [1-3]. Four unidirectional basic subnets - line, merge, divide and intersection - for the AGVS have been established in [2]. The AGVS Petrinet structure, the corresponding AGVS Petri-net properties (safeness, boundedness, strict conservation, reachability and liveness), the way to embed those properties into the nets, and the way to identify the robustness of the nets have been discussed thoroughly in [3]. In this paper, based on previous research results, more different types of subnets for both unior bi-directional systems will be established, and the robustness of every net will be proved. For the purpose of simplifying the union steps, the submodel modulised concept is adopted in this paper. Therefore, the complete model can be developed straightforwardly by connecting the input part of a subnet with the output part of another subnet. The modulised floorpath net is thus given as the name instead of the subnet.

\section{Reviews of AGVS and Petri-nets Structures}

Petri nets were chosen as the modelling language for the development of the AGVS modelling tool. The AGVS robustness properties and its corresponding Petri-net structure and properties will be reviewed here.

\subsection{Petri Net Review [3,4]}

A Petri net may have safeness, boundedness, strict conservation, reachability and liveness properties. By observing its reachability tree, one can examine the safeness, boundedness and conservation properties of a net. It has been proved that the reachability problem is equivalent to the liveness problem. Hence, if a net is live, all the paths of the net are reachable. Although the reachability tree does not necessarily always 
contain enough information to solve the liveness problem, it is the case that the net is live if all separate subtrees are associated with markings which are the same as that of the frontier node, and every transition has been fired at least once. By the use of the incident matrix equation, if every element of the weighting vector is 1 , the Petri net is strictly conservative. If a net is conservative, the net must be bounded.

\subsection{AGVS and its Petri Nets [3]}

According to [3], in order to keep an AGVS functioning well, the AGVS should have four properties, system collision free, constant numbers of vehicles and traffic control signals, paths reachable and system deadlock free, which correspond respectively to Petri-net properties, safeness, boundedness, strict conservation, reachability and liveness. Therefore, the safeness, boundedness, strict conservation, reachability and liveness are the necessary Petri-net properties in the AGVS floor-path net. For the implementation of the zone control function by Petri nets, zones are distiguished as physical zones, pseudo-zones and composite pseudo-zones.

An AGVS floor-path net is composed of a flow-path net and a control-loop net. The flow-path net reflects the physical flow-path layout, and the control-loop net represents the traffic control components. In the flow-path net, each place represents either a physical zone, a pseudo-zone, or a composite pseudo-zone. The direction of the traffic flow is represented by the directed arc. Tokens in places represent vehicles. Transitions represent permissions for vehicles to move from one place to another place. Each place in the control-loop net represents a control node. Tokens in each place represent signals or commands. Directed arcs are used to direct the flow of signals or commands. There is no other set of transitions which belongs to the control-loop net itself. Both the control-loop net and the flow-path net share the same transitions. To fulfil the zone control function, the transition can be fired only when the input places of the flowpath net and control-loop net have enough tokens and the output place of the flow-path net is empty. The complete net can be obtained by placing the control-loop net over the flowpath net.

In an AGVS floor-path net:

1. The flow-path net is not a closed-system net, while the control-loop net is.

2. The necessary properties can be embeded into the nets by adding extra places at proper locations and checked by the use of its reachability tree or incident matrix equations.

3. If those properties exist in both the flow-path net and the control-loop nets, they also exist in the complete net.

\section{Modulised Floor-Path Nets}

In this section, several basic floor-path nets will be established. For the purpose of simplifying the union procedure, in this study, not only is the modulised concept adopted, but also the interface between two nets such as the input of a net and/or the output of a net is clearly defined, so that, at the union stage, one can directly connect the output (or input) zone of a net with the input (or output) zone of the other net. Both input and output zones belong to the physical zone.

Since every flow-path net represents a feature of the AGVS floor-path structure, it is not necessary to be a closed system. In order to save the information in the net, the control-loop net is always a closed system. The modulised floor-path net is therefore not a closed system. Because of this, all the property analyses discussed in this paper are within the input and output zones of the net. The discussion of the liveness property for those nets has to be modified. For any flow-path net, if a vehicle at the input zone can arrive safely at one of the output zones, and, as long as that vehicle is removed from the output zone, any other vehicle at an input zone can always move to one of the output zones, and if, by continuously removing vehicles from output zones, it can keep any vehicle (if there is at least one) at an input zone moving to the output zone, it is said that the net has the potential to be live. Therefore, in this paper, if the reachability tree of a non-closed net shows that every individual vehicle at the input zone can move to the output zone, it is said that the net is live within input and output zones.

For the purpose of distinguishing the flow-path net and control-loop net, solid lines are used to draw the flow-path net, and dashed lines are used to draw the control-loop net. Big dark circles represent tokens in the flow-path net, and small dark circles represent tokens in the control-loop net. For analysis purposes, tokens representing vehicles will be placed at the input zones of the flow-path net in the paper. In fact, at the floor-level design stage, vehicles are not there. Hence, in any modulised floor-path net, big circle tokens should be eliminated once the anlayses have been done, while small circle tokens should always be there to ensure the traffic control functions.

\subsection{Uni-directional System}

Most of the existing AGVSs are uni-directional. This is because the control of the system is much simpler. Vehicles in the system move in direction, which results in the workpart transportation sometimes being very inefficient if the delivery station happens to be next to the pick-up station, but in the opposite direction to the traffic flow. Usually, additional spur track added in a proper way solve the problem. In the following, based on the uni-directional system, several modulised floorpath nets will be established and analysed one by one.

\section{Line}

The line structure connects one zone with another (see Fig. 1). $Z_{1}$ is the input zone and $Z_{2}$ is the output zone. The flow-

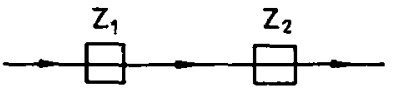

Fig. 1. The line floor-path structure. 


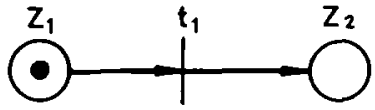

Fig. 2. The line net.

path net of the line structure which consists of two places. $Z_{1}$ and $Z_{2}$, and one transition, $t_{1}$, is established in Fig. 2. Since it is so simple. it does not need any control node. Therefore, the control-loop net is not necessary.

For the analysis, a token which represents a vehicle is placed in the input zone $Z_{1}$. The initial marking of the net is $\left(Z_{1}, Z_{2}\right)=(1,0)$. At this moment, $t_{1}$ is fireable. After $t_{1}$ is fired, the marking becomes $(0,1)$. It is mentioned above that the property analyses are done within input and output zones. Hence, all the possible states of the net have been presented. The total number of vehicles for each possible state is 1 , and the maximum tokens in any place is 1 . Therefore, the net is safe as well as strictly conservative. In fact, because of the modified transition firing rule, tokens in the flow-path net can never exceed 1 . Hence, the safeness property is always true in any flow-path net. From now on, the safeness property will not be checked again. As for the reachability property, in this net, there are only two elements, $(1,0)$ and $(0,1)$, in the reachability set. and only two segments of paths. Hence. every path in the net is reachable. It has been mentioned above that the reachability problem is equivalent to the liveness problem. In the rest of the paper, as long as the net is live. the reachability property is true too. As for the liveness property, because a vehicle at $Z_{1}$ can always arrive at $Z_{2}$ if $t_{1}$ is fired, hence, the net is live within input and output zones.

Although the line net is so simple, it is the most common net in the AGVS. The property analysis of the line net is as important as those of other nets.

\section{Divide}

As shown in Fig. 3, in a divide structure, a vehicle can move from zone $Z_{1}$ to either zone $Z_{2}$ or $Z_{3}$. In this structure, there is no need to have control functions other than the zone control function. Therefore, the control-loop net is not necessary for this structure. According to the structure, the divide flow-path net is established in Fig. 4. There are three places $Z_{1}, Z_{2}$ and $Z_{3}$ representing the three physical zones, and two transitions $t_{1}$ and $t_{2} . Z_{1}$ is the input zone and $Z_{2}$ and $Z_{3}$ are the output zones of the net. For analysis purposes, a

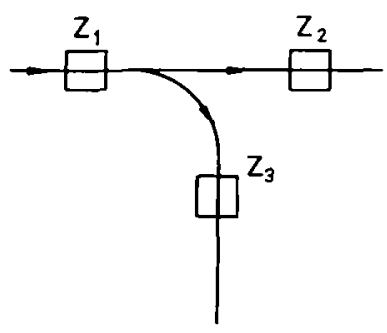

Fig. 3. The divide floor-path structure.

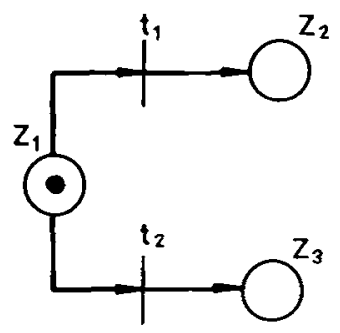

Fig. 4. The divide net.

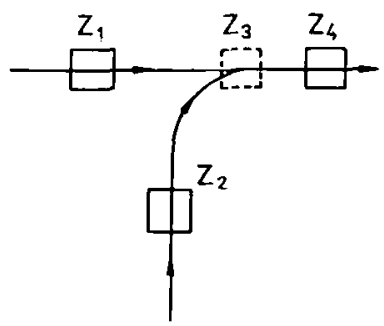

Fig. 5. The merge floor-path structure.

token is placed in $Z_{1}$. A letter $\mathrm{D}$ in a box is the symbol of the modulised divide net. It is expected that a desired net will be automatically built at a desired location if the user places the symbol of the desired net at the desired location.

In Fig. 4 , the initial marking is $(1,0,0)$. After $t_{1}$ or $t_{2}$ is fired, the new marking is $(0,1,0)$ or $(0,0,1)$. Again, property analyses are done between input and output zones. Therefore, conclusions can be made regarding the properties. Since the total number of tokens is 1 at any state, the net is strictly conservative. The vehicle at the input zones can arrive at either of the output zones. Hence, the net is live within the input and output zones.

\section{Merge}

A modified merge structure has been discussed in [3]. Here, the merge structure wil be studied again within the input and output zones.

Fig. 5 is the physical merge structure; two lanes merge into one lane. $Z_{1}$ and $Z_{2}$ are the input zones, and $Z_{4}$ is the output zone. The non-closed flow-path net is shown in Fig. 6. Since the control-loop net has been analysed in [3], it is not necessary to redraw and analyse it here again.

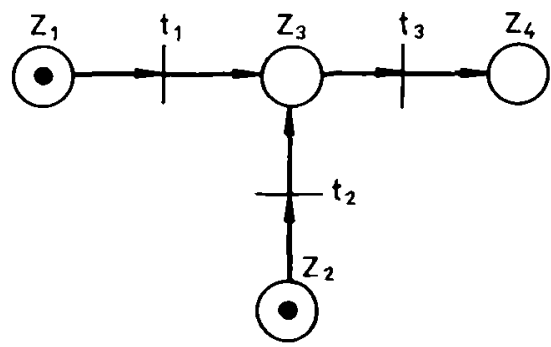

Fig. 6. The merge flow-path net. 
The incident matrix of the flow-path net is:

$$
N_{\mathrm{f}}=t_{t_{2}}^{t_{3}}\left[\begin{array}{rrrr}
Z_{1} & Z_{2} & Z_{3} & Z_{4} \\
-1 & 0 & 1 & 0 \\
0 & -1 & 1 & 0 \\
0 & 0 & -1 & 1
\end{array}\right]
$$

There exists a weighting vector $W_{\mathrm{f}}=(1,1,1,1)^{\mathrm{T}}$ such that $N_{\mathrm{f}} \cdot W_{\mathrm{f}}=0$. Thus, the flow-path net is strictly conservative.

In Fig. 6, the initial marking is $(1,1,0,0)$. Once $t_{1}$ (or $t_{2}$ ) is fired, the new marking is $(0,1,1,0)$ (or $(1,0,1,0)$ ). Since $Z_{3}$ is a pseudo-zone, $t_{3}$ has to be fired immediately after the firing of $t_{1}$ (or $t_{2}$ ). After $t_{3}$ is fired, the marking becomes $(0,1,0,1)$ (or $(1,0,0,1)$ ). since each individual vehicle at the input zone can safely arrive at the output zone, the net is live within the input and output zones.

To combine the flow-path and control-loop nets, the complete merge net can be obtained as is shown in Fig. 7 An "M" in a box is its symbol. the places $Z_{3}$ and $C_{2}$ are piled one upon another. From Fig. 7, one can see that:

1. When $t_{1}$ is fired, the token in $C_{1}$ is moved to $C_{2}$. Hence, $t_{2}$ cannot be fired. This guarantees that the vehicle from $Z_{1}$ will not collide with the vehicle from $Z_{2}$. Because $Z_{3}$ is a pseudo-zone, the vehicle from $Z_{1}$ cannot stay (pseudozones do not have enough space to hold a vehicle) at $Z_{3}$ and has to leave immediately, $t_{3}$ has to be fired immediately after $t_{1}$ is fired. A vehicle from $Z_{1}$ thus enters $Z_{4}$, and $C_{2}$ returns the token to $C_{1}$ in order to reset the control status of the net.

2. After the vehicle from $Z_{1}$ enters $Z_{4}, t_{2}$ satisfies the firing rules. However, since the immediate physical zone $Z_{\downarrow}$ is not free, $t_{2}$ is not fireable. The vehicle at $Z_{2}$ has to wait until the vehicle at $Z_{4}$ leaves the net. As long as the vehicle at $Z_{d}$ leaves the net, the net is live again.

3. Similarly, at this moment, if a vehicle enters $Z_{1}$, it has to wait until the net is live again.

Since both the flow-path net and the control-loop net satisfy the robustness properties, the complete net also has properties. For the purpose of supporting the statement "the net is live within the input and output zones", the extended reachability tree of the net is drawn in Fig. 8. In Fig. 8, those net states above the dashed line are the states within the input and output zones. They indicate that each individual vehicle at the input zone can arrive at the output zone. Those net states below the dash line are the future states where the output

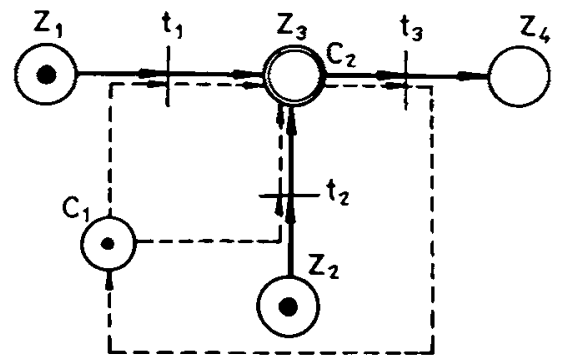

Fig. 7. The merge net.

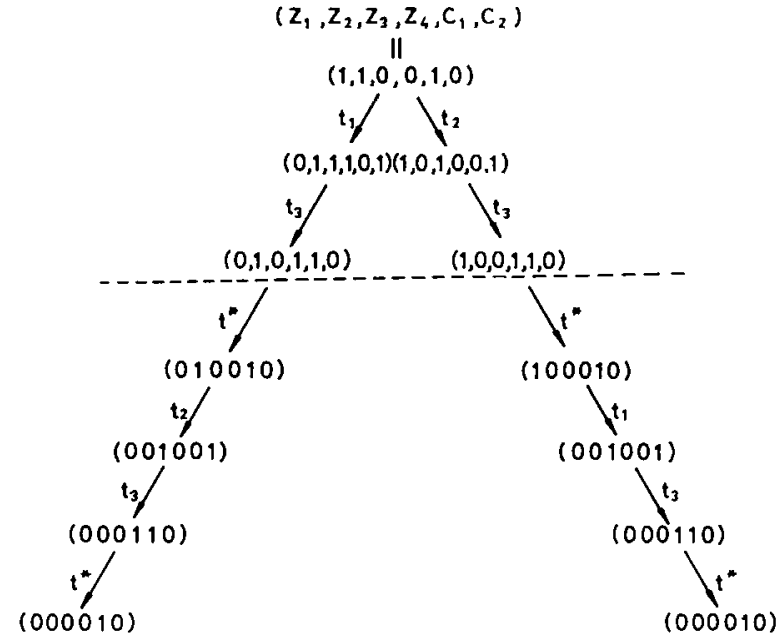

Fig. 8. The extended reachability tree of the merge net.

zone of the net is connected with the input zone of another net. The firing of the first $t^{*}$ in Fig. 8 represents a vehicle leaving the net from the output zone. At this moment, $t_{2}$ can be fired, and $t_{3}$ has to be fired immediately after $t_{2}$ is fired. Then, if the second $t^{*}$ is fired, the last vehicle in the system leaves the net. Finally, there is only a control signal left in the net. The marking of the control-loop net returns to the original state, and the flow-path net is now ready for another vehicle to pass through.

Thus, the definition of the liveness within input and output zones for those open systems is feasible, and the modulised merge net is robust under the definition.

\section{Intersection}

Fig. 9 is the intersection floor-path structure. There are four physical zones, $Z_{1}, Z_{2}, Z_{3}$ and $Z_{4}$, and a composite pseudozone $Z_{5} . Z_{1}$ and $Z_{2}$ are the input zones, and $Z_{3}$ and $Z_{4}$ are the output zones. The traffic control at the intersection has to include the following functions:

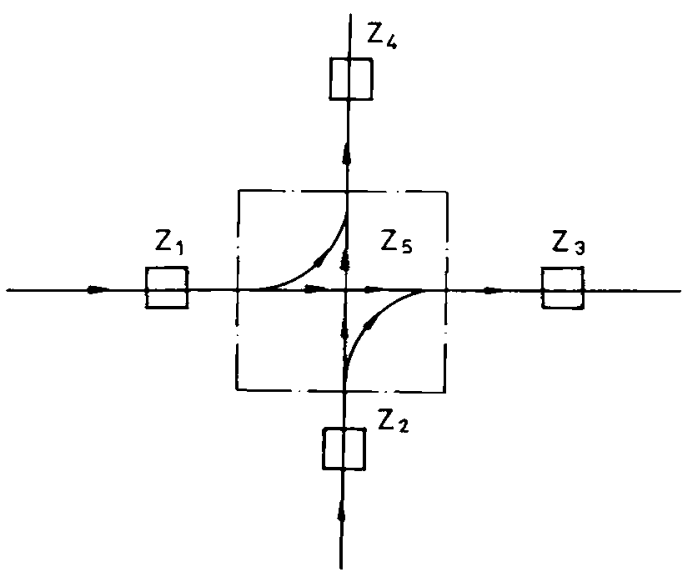

Fig. 9. The intersection floor-path structure. 


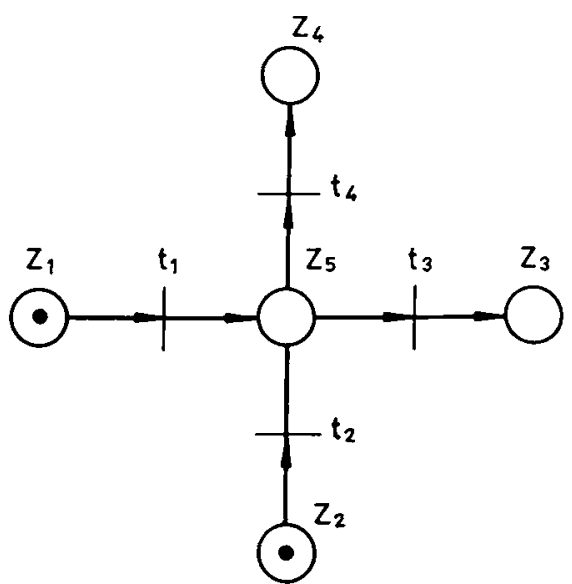

Fig. 10. The intersection flow-path net.

1. To control the movements of vehicles from $Z_{1}$ and $Z_{2}$ to $Z_{3}$ or $Z_{4}$

2. To prevent that the vehicle from $Z_{1}$ to $Z_{3}$ bumping into another vehicle from $Z_{2}$ to $Z_{4}$

3. To prevent a vehicle from $Z_{1}$ to $Z_{4}$ passing the vehicle from $Z_{2}$ to $Z_{3}$ so closely that they hit each other.

Fig. 10 is the flow-path net of the intersection. The incident matrix of the net is:

$$
N_{\mathrm{f}}=\left[\begin{array}{rrrrrr} 
& Z_{1} & Z_{2} & Z_{3} & Z_{4} & Z_{5} \\
t_{2} & -1 & 0 & 0 & 0 & 1 \\
t_{3} & 0 & 0 & 1 & 0 & -1 \\
t_{4} & 0 & 0 & 0 & 1 & -1
\end{array}\right]
$$

There exists a weighting vector $W_{\mathrm{f}}=(1,1,1,1,1)^{\mathrm{T}}$ such that $N_{\mathrm{f}} \cdot W_{\mathrm{f}}=0$. Hence, the flow-path net is strictly conservative and bounded. The liveness problem of the flow-path net is the same as that of the complete net, and will be discussed after the complete net is established.

Fig. 11 is the control-loop net of the intersection structure. Since the three control functions mentioned above have to be embeded in the net, two control places $C_{1}$ and $C_{2}$ are used to achieve them. A token in $C_{1}$ represents the right of

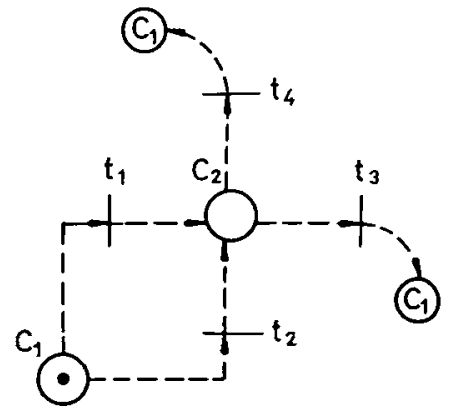

Fig. 11. The intersection control-loop net.

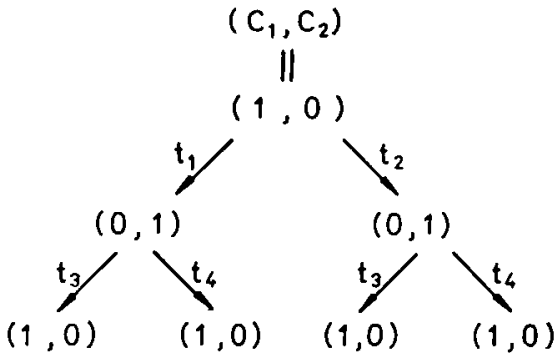

Fig. 12. The reachability tree of the intersection control-loop net.

way at the intersection. Place $C_{2}$ is used to preserve the token so that the traffic control signals in the net will not be lost.

The incident matrix of the control-loop net is:

$$
N_{c}=\left[\begin{array}{rrr} 
& C_{1} & C_{2} \\
t_{1} & -1 & 1 \\
t_{2} & -1 & 1 \\
t_{3} & 1 & -1 \\
t_{4} & 1 & -1
\end{array}\right]
$$

There exists a weighting vector $W_{\mathrm{c}}=(1,1)^{\mathrm{T}}$ such that $N_{\mathrm{c}} \cdot W_{\mathrm{c}}=0$. Hence, the net is strictly conservative and also bounded

Fig. 12 is the reachability tree of the control-loop net. The initial marking is $(1,0)$. After a series of transition firings, the marking of each subtree returns the initial marking, and every transition in the net has been fired at least once. Therefore, the control-loop net is live.

By placing the control-loop net over the flow-path net, the complete intersection net is thus established (see Fig. 13). A letter "I" in a box is the symbol of the intersection net. Since both the flow-path and control-loop nets are strictly conservative and bounded, the complete net is also.

Fig. 14 is the reachability tree of the intersection net. It shows that each individual vehicle can arrive at each of the output zones respectively. Therefore, the net is live within the input and output zones.

Thus, the modulised intersection net is robust.

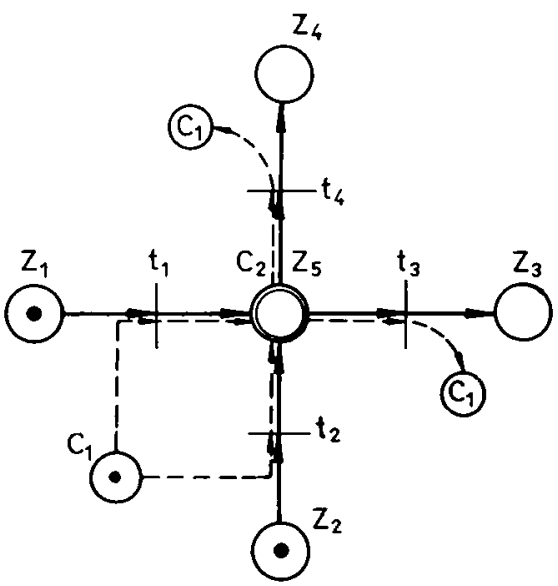

Fig. 13. The intersection net. 


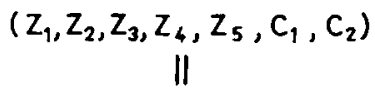

(1100010)

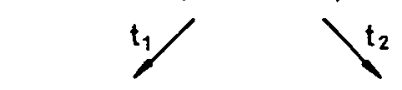

(0100101)

(1000101)

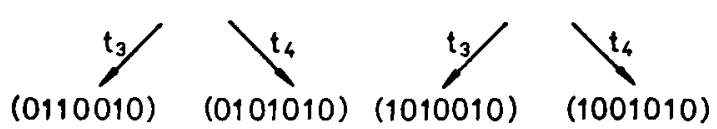

Fig. 14. The reachability tree of the merge net.

\subsection{Bi-directional System}

In the design of a bi-directional guide-path system, three alternatives can be adopted. These alternatives include the following [5]:

1. Have parallel wire tracks with reverse orientation on each aisle.

2. Have a single switchable wire-track on each aisle. The switching of the guide-path is dependent on the flow demand.

3. Have a mixed guide-path that is comprised of both unidirectional and bi-directional aisles, with bi-directional flow paths allowed only on selected aisles.

The alternative 2 is the uni-directional system for a short time period. The floor-path model can be built by the union of the uni-directional nets. The bi-directional path of the alternative 3 are usually built in those areas where only one vehicle is allowed in and out at one time. The alternative 1 is the most common one. In this paper, the alternative 1 is adopted to build the bi-directional AGVS.

Except at points of intersection or merge, the guide-path system of the bi-directional system is essentially uni-directional. With sufficient clearance space left between parallel tracks. there is virtually no interference between vehicles on the same aisle when travelling in opposite directions. Therefore, merge and intersection are the two main structures discussed in this section.

It is almost impossible to illustrate all the possible bidirectional system structures here. In the following, several merge and intersection nets are established one by one. Hopefully, one can build a bi-directional AGVS model by the union of the nets developed here. Since these nets are much more complex when compared with the uni-directional system, a capital "C" which is the acronym of "complex" is used before the names of the structures in order to distinguish them from the uni-directional structures.

\section{Rotation cycles}

Structures in the bi-directional system are relatively complex when compared with those of the uni-directional system. Therefore, the reachability tree is sometimes too large to be illustrated in the paper. Instead of drawing the reachability tree, a "rotation cycle" concept is created. The concept of the rotation cycle is based on the reachability tree. In a closed-loop net, for each sub-branch of the reachability tree, a sequence of firing transitions could be found. If the net is live, the last marking of each sub-branch must be the initial marking. Hence, if one more transition is fired in each subbranch, the fired transition must be the first transition of the sequence of firing transitions. By putting the first transition as the last of each sequence of firing transitions, several transition cycles can thus be formed. Each cycle can be mapped to a sub-branch of the reachability tree, each subbranch of the reachability tree can form a cycle, and every transition of the net should be included in one or more cycles. Among those cycles, some are long enough to include other cycles, and some are not. Those cycles which are too short to include another cycle are named "rotation cycles".

Definition. A rotation cycle in an AGVS net is the shortest sequence of transitions $\sigma=t_{i_{1}} t_{j_{2}} \ldots t_{j_{k}}$ such that for each $t_{j_{r}}$ and $t_{j_{r+1}}$ in the sequence there is a place $p_{i_{r}}$ with $p_{i_{r}} \in O\left(t_{j_{r}}\right)$ and $p_{i_{r}} \in I\left(t_{j_{r+1}}\right), t_{j_{1}}=t_{j_{k}}$, and $\mu^{\prime}=\delta\left(\mu, t_{j_{1}}\right)=\delta(\mu, \sigma)$, where $\mu$ is the initial marking of the net.

A rotation cycle is thus the shortest closed path from a transition back to that same transition. A rotation cycle can be proved to have conservation, boundedness, reachability and liveness properties. If a net can be decomposed into several rotation cycles, and every transition in the net has been included in one or more rotation cycles, the net is live.

\section{C-Line}

Observing the line structure of the bi-directional system, except that there are two flows with reverse orientation, each of the fows is exactly identical to that in the uni-directional line structure. Therefore, the modulised $C$-line net can be established by the union of the two uni-directional line nets.

\section{C-Merge 1}

Fig. 15 is one of merge structures for the bi-directional AGVS. In the figure, places $Z_{6}$ and $Z_{7}$ are pseudo-places which are used to prevent vehicle from $Z_{1}$ to $Z_{5}$ and vehicle from $Z_{2}$ to $Z_{4}$ or $Z_{5}$ from bumping against each other. There are two input zones, $Z_{1}$ and $Z_{2}$, and three output zones, $Z_{3}, Z_{4}$ and $Z_{5}$. The flow-path net of the C-Merge $I$ is shown in the Fig. 16. For analysis purposes, vehicles are placed in the two input zones.

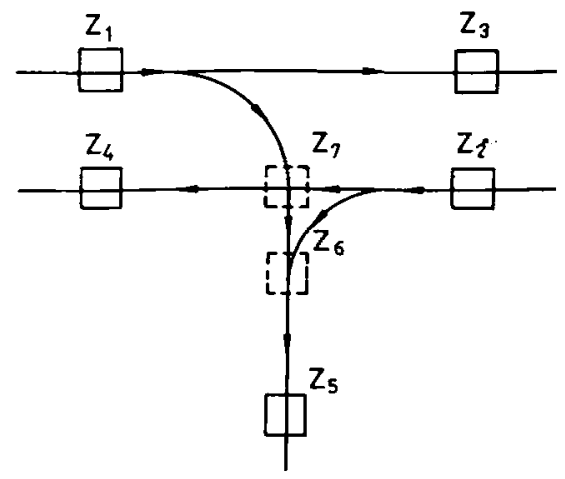

Fig. 15. The C-Merge I structure. 


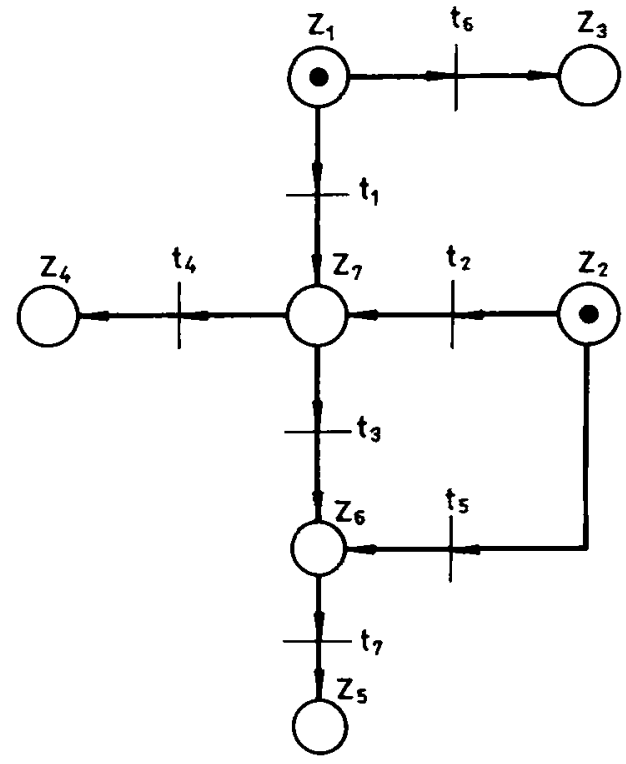

Fig. 16. The flow-path net of the C-Merge I structure.

In a similar way to the conservation analysis for the unidirectional net, the incident matrix $N_{\mathrm{f}}$ of the flow-path net can be obtained, and also it can be proved that there exists a weighting vector $W_{1}=(1,1,1,1,1,1,1)^{\mathrm{T}}$ such that $N_{\mathrm{f}} \cdot W_{\mathrm{f}}=0$. Because every element in $W_{\mathrm{f}}$ is 1 , the flow-path net is strictly conservative and bounded. By observing the flow-path net, it can be seen that the individual vehicle at the input zone can arrive at the respective output zone and the net is live within input and output zones.

Fig. 17 is the control-loop net of this C-Merge I structure. The token in place $C_{1}$ is used to prevent vehicles entering $Z_{6}$ or $Z_{7}$ at the same time. The token in place $C_{2}$ is used to prevent vehicles from $Z_{1}$ entering $Z_{4}$ (a vehicle cannot usually make a turn of more than $90^{\circ}$ ). $C_{3}$ and $C_{4}$ are used to preserve tokens in the net. By the use of incident matrix equations, it can be proved that there exists a weighting vector $W_{c}=(1,1,1,1)^{\mathrm{T}}$ such that $N_{\mathrm{c}} \cdot W_{\mathrm{c}}=0$. Hence, the net is strictly conservative and bounded.

Since the reachability tree of the control-loop net is relatively large instead of drawing the reachability tree, the "rotation cycle" concept mentioned above is used to analyse the liveness property. From Fig. 17, it can be found that there are exactly three rotation cycles in the net. These rotation cycles are:

1. $t_{5} t_{7} t_{5}$

2. $t_{2} t_{4} t_{2}$

3. $t_{1} t_{3} t_{7} t_{1}$

Every transition in the control-loop net has ben inlcuded in one or more rotation cycles, hence, the control-loop net is live. Note: Transition $t_{6}$ is not in Fig. 17.

Fig. 18 is the complete net of the C-Merge I structure. A symbol "C-M I" in a box represents the modulised C-Merge I net. Since both flow-path and control-loop nets are robust, the complete net is also robust.

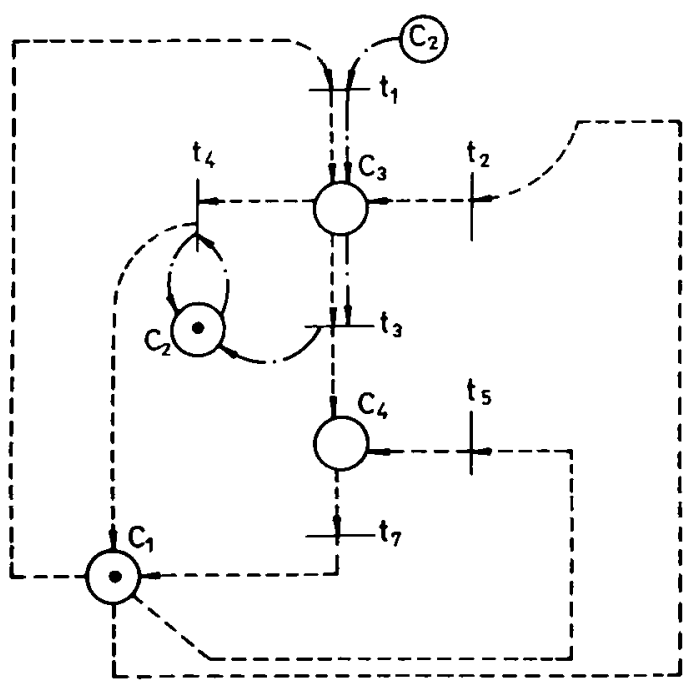

$-----C_{1}$ control line $-.-\cdot-c_{2}$ control line

Fig. 17. The control-loop net of the C-Merge I structure.

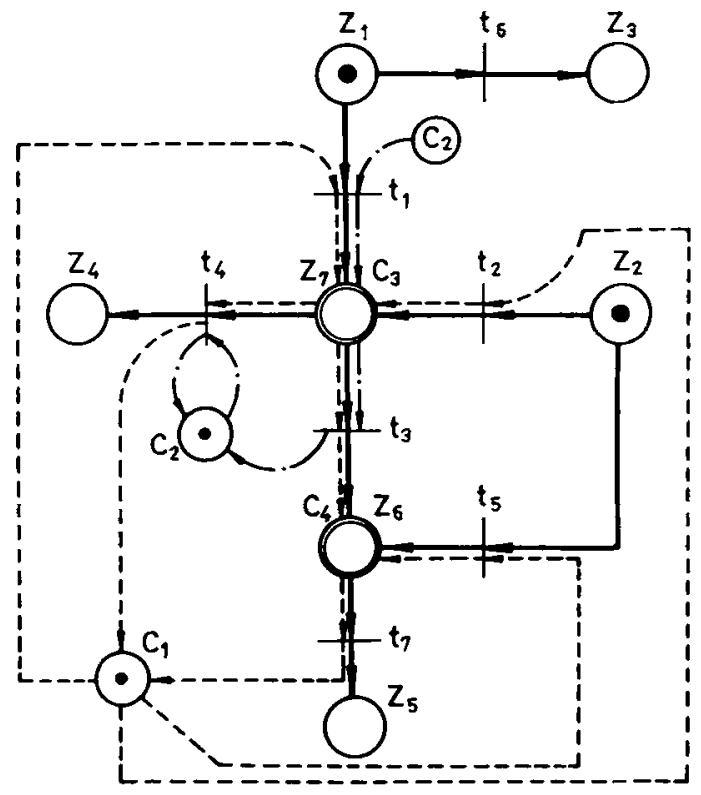

Fig. 18. The C-Merge I net.

\section{C-Merge II}

Fig. 19 is another merge structure for the bi-directional AGVS. In the figure, $Z_{6}$ is a composite pseudo-zone, and $Z_{7}$ is a pseudo-zone. There are three input zones, $Z_{1}, Z_{2}$ and $Z_{3}$, and two output zones, $Z_{4}$ and $Z_{5}$. Vehicles from $Z_{1}$ can move to $Z_{4}$ and $Z_{5}$, vehicles from $Z_{2}$ can move to $Z_{4}$, and 


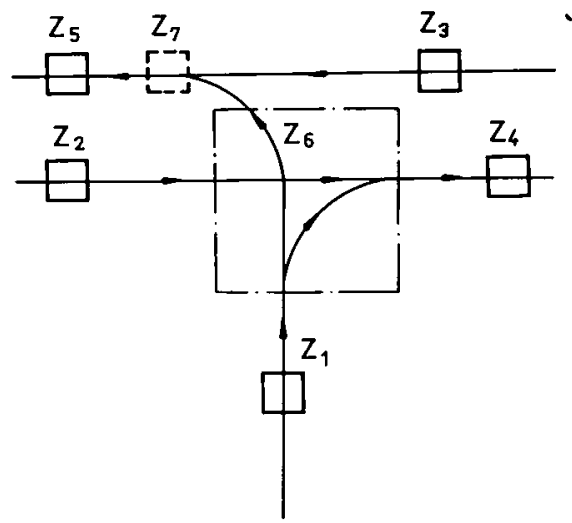

Fig. 19. The C-Merge II structure.

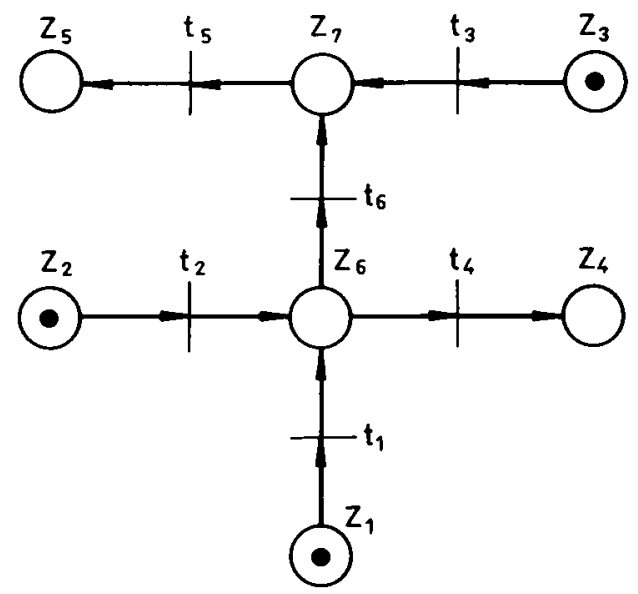

Fig. 20. The flow-path net of the C-Merge II structure. vehicles from $Z_{3}$ can move to $Z_{5}$. The possible traffic problems of this structure are:

1. $Z_{1}$ vehicle wants to move to $Z_{5}$ while $Z_{2}$ vehicle wants to move to $Z_{4}$.

2. $Z_{1}$ vehicle wants to move to $Z_{4}$ while $Z_{2}$ vehicle wants to move to $Z_{4}$ too.

3. $Z_{1}$ vehicle wants to move to $Z_{5}$ while $Z_{3}$ vehicle wants to move to $Z_{5}$ too.

Therefore, several, control nodes have to be added into the structure.

Fig. 20 is the flow-path net of this structure. By the use of incident matrix equations, it can be proved that the flow-path net is strictly conservative and bounded. By observing the flow-path net, the property - the liveness within input and output zones - can be seen. The flow-path net is robust.

Fig. 21 is the control-loop net of this structure. A token in $C_{1}$ (or $C_{2}$ ) is used to prevent more than one vehicles entering $Z_{6}$ (or $Z_{7}$ ) at the same time. The $C_{3}$ token is used to restrict the movement of the $Z_{2}$ vehicle so that it does not go to $Z_{1}$. The control place $C_{6}$ is used to return tokens to $C_{2}$ or $C_{3}$. A small circle instead of an arrowhead at the transition $t_{7}$ or $t_{8}$ is called an inhibitor arc [4]. This notation is borrowed from switching theory where the small circle means "NOT". The firing rule is changed as follows: a transition is enabled when tokens are in all of its inputs and zero tokens are in all of its inhibitor inputs. The transition fires by removing tokens from all of its inputs.

Fig. 19 shows that the vehicle from $Z_{1}$ to $Z_{4}$ should not interfere with the vehicle from $Z_{3}$ to $Z_{5}$. However, Fig. 21 shows that the firings of transition $t_{3}$ and $t_{1}$ are in conflict. This means that when a vehicle moves from $Z_{1}$ to $Z_{4}$ and another vehicle moves from $Z_{3}$ to $Z_{5}$ they may interfere with each other. This can be improved by the decomposition of $Z_{6}$ into two pseudo-zones. However, the control-loop net becomes more complex. This is a trade-off between cost and efficiency.
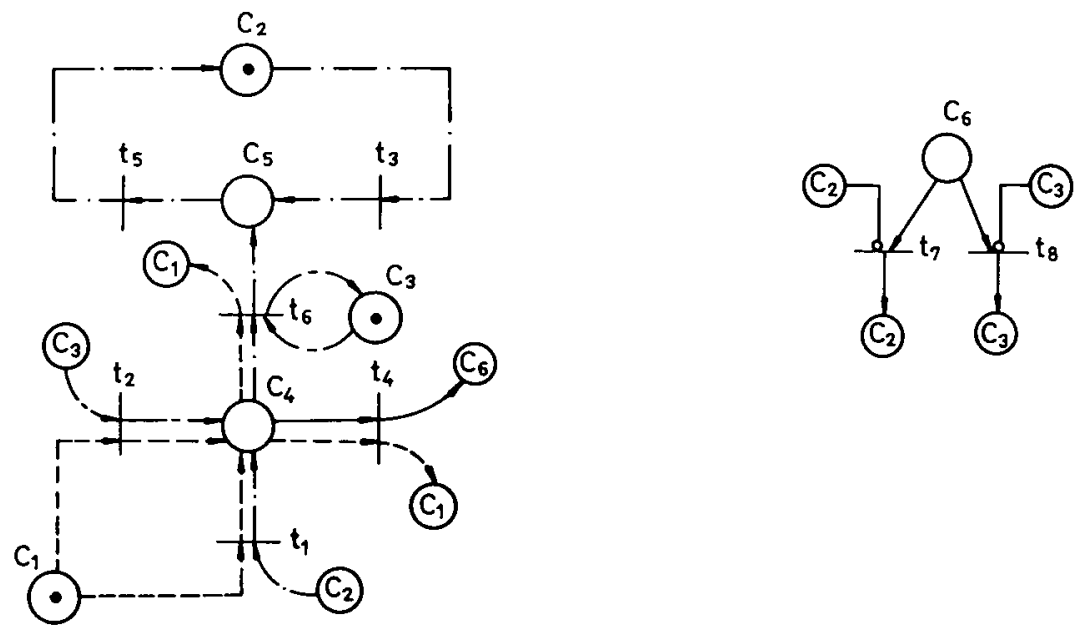

Fig. 21. The control-loop net of the C-Merge III structure. 


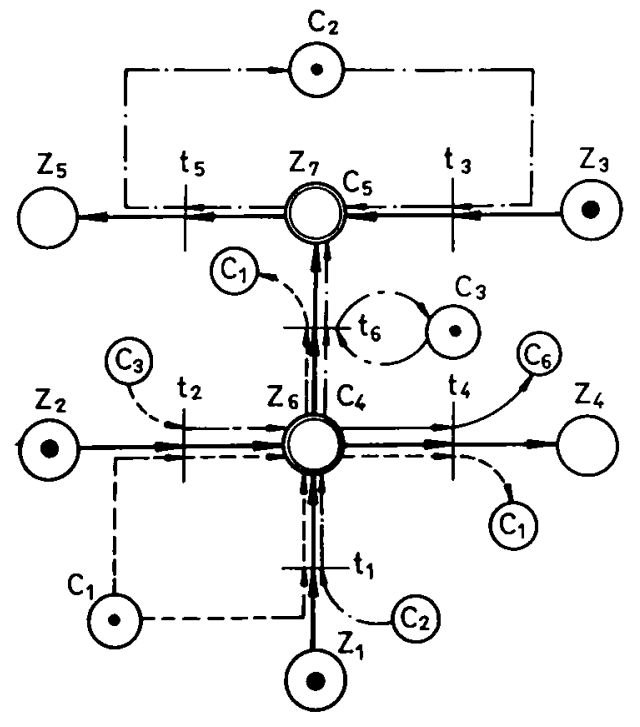

Fig. 22. The C-Merge II net.

By the use of incident matrix equations, it can be proved that the net is strictly conservative and bounded. In Fig. 21, there are four rotation cycles in the net. They are: $t_{3} t_{5} t_{3}$, $t_{1} t_{6} t_{5} t_{1}, t_{1} t_{4} t_{7} t_{1}$ and $t_{2} t_{4} t_{8} t_{2}$. Since the control-loop net can be decomposed into four rotation cycles, and every transition in the net has been included in at least one rotation cycle, therefore, the net is live.

Fig. 22 is the complete net of the C-Merge I structure. A symbol "C-M II" in a box is given to represent the net. Since both the flow-path and control-loop nets are robust, the complete net is also robust.

\section{C-Merge $/ /$}

Fig. 23 is a more complete merge structure for the bidirectional system. In the figure, places $Z_{7}, Z_{8}$ and $Z_{9}$ are pseudo-zones, and $Z_{10}$ is a composite pseudo-zone. There are three input zones, $Z_{1}, Z_{2}$ and $Z_{3}$, and three output zones, $Z_{4}$, $Z_{5}$ and $Z_{6}$. The possible traffic problems of this structure are:

1. $Z_{1}$ vehicle wants to move to $Z_{6}$ while $Z_{2}$ vehicle wants to move to $Z_{5}$ or $Z_{6}$.

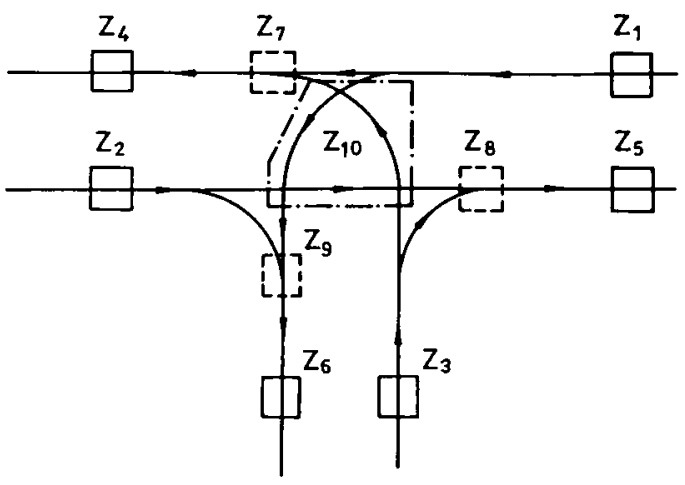

Fig. 23. The C-Merge III structure.
2. $Z_{1}$ vehicle wants to move to $Z_{4}$ or $Z_{6}$ while $Z_{3}$ vehicle wants to move to $Z_{4}$.

3. $Z_{2}$ vehicle wants to move to $Z_{6}$ while $Z_{3}$ vehicle wants to move to $Z_{4}$ or $Z_{5}$.

Therefore, a control-loop net is necessary.

Fig. 24 is the complete "C-Merge III" net which is the combination of the flow-path net (solid line portion) and the control-loop net (dash line portion). A symbol "C-M III" in a box is given to represent the net. The strict conservation property of both the flow-path net and control-loop net of this structure can be proved by incident matrix equations. As for the liveness property, the reachability tree or the rotation cycle concept can be used to prove it. Since both the flowpath and control-loop nets can be proved to be robust, the complete net is also robust.

\section{Inter-Merge}

Fig. 25 is one of the possible structures for the bi-directional system. It is named as the Inter-Merge structure. There are three input zones, $Z_{1}, Z_{2}$ and $Z_{3}$, and three output zones, $Z_{4}, Z_{5}$. and $Z_{6}$. For the purpose of simplifying the control problem, in the figure, only two composite pseudo-zones, $Z_{7}$ and $Z_{8}$ are used. In a similar way to the C-Merge II structure, the proposed structure of Fig. 25 is a trade-off between cost and efficiency. When the vehicle is moving from $Z_{1}$ through $Z_{7}$ to $Z_{6}$. physically, the vehicle at $Z_{2}$ can move to $Z_{4}$ or $Z_{5}$. However, technically, the right (the token) of way will not be issued to the vehicle at $Z_{2}$ until the former vehicle arrives at $Z_{n}$. The possible traffic problems of this structure are:

1. $Z_{1}$ vehicle wants to move to $Z_{4}$ or $Z_{5}$, and at the same time $Z_{2}$ vehicle wants to move to $Z_{4}$ or $Z_{5}$ too.

2. $Z_{1}$ vehicle wants to move to $Z_{4}$ or $Z_{5}$ while $Z_{3}$ vehicle wants to move to $Z_{6}$.

3. $Z_{1}$ and $Z_{3}$ vehicles want to move to $Z_{6}$ at the same time.

Therefore, the control-loop net is necessary.

Property analyses can be carried out on the flow-path net and control-loop net. The analysis results indicate that both nets are robust. Hence, the complete net is also robust. The modulised "Inter-Merge" net is shown in Fig. 26. A symbol "I-M" is given to represent this net.

\section{$C-I-M$}

Fig. 27 is the floor-path layout of the C-I-M structure. The "C-I-M", where " $C$ " is the acronym of "complex, "I" is "intersection", and " $M$ " is merge, represents a two-way intersection with a merge traffic-flow structure. There are four input zones (from $Z_{1}$ to $Z_{4}$ ) and four output zones (from $Z_{5}$ to $Z_{4}$ ). This is a very complex traffic structure. Many traffic problems could occur in this structure. In the figure, $Z_{4}, Z_{11}, Z_{11}$ and $Z_{12}$ are the four pseudo-zones. Because there are too many possible traffic problems, a composite pseudo-zone $Z_{13}$ is used to control the four-way traffic in the middle. Since the net is too complex to be described and analysed here, only the complete C-I-M net is shown in Fig. 28. From this, it can be seen how difficult it is to adopt a structure like this. 


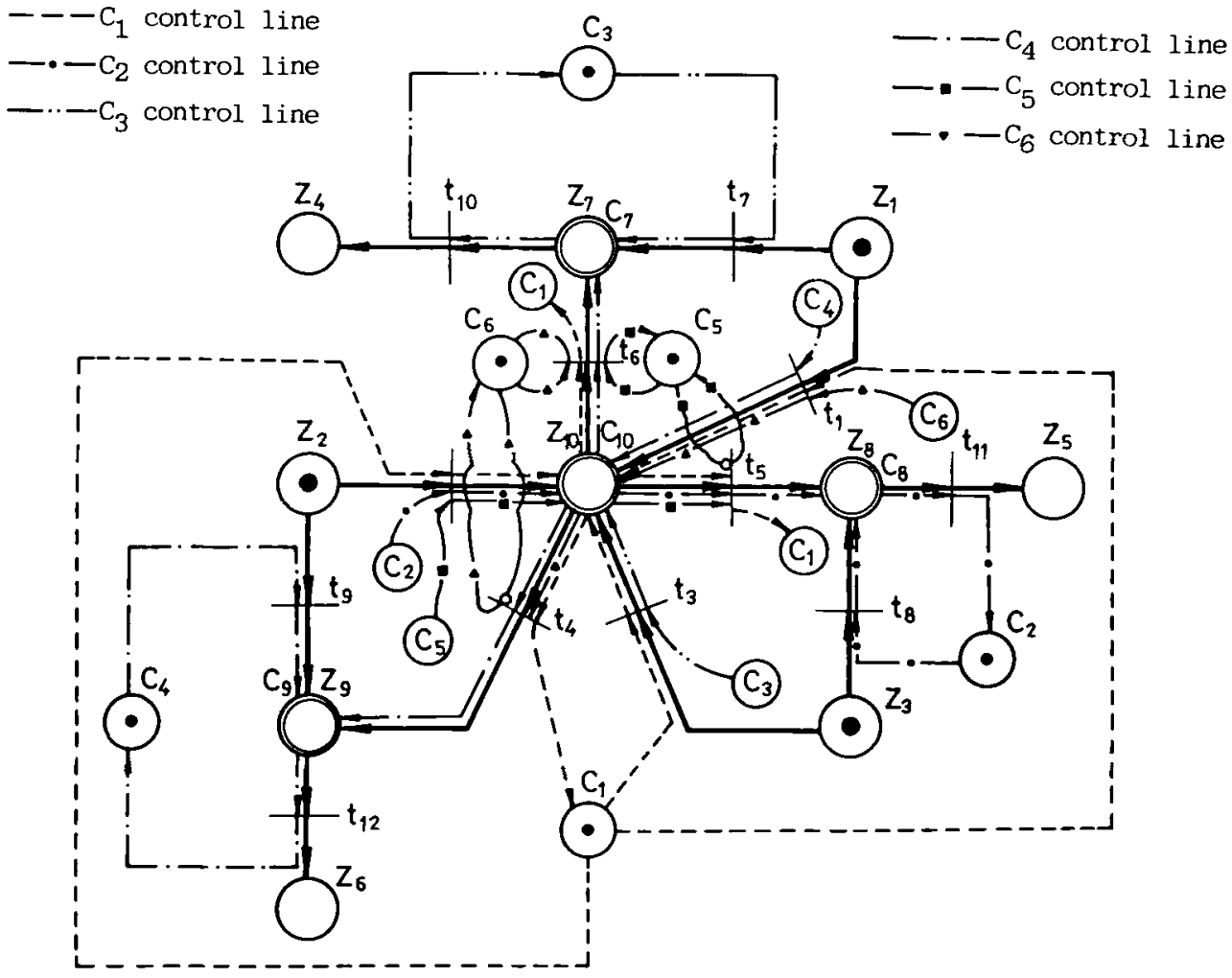

Fig. 24. The C-Merge III net.

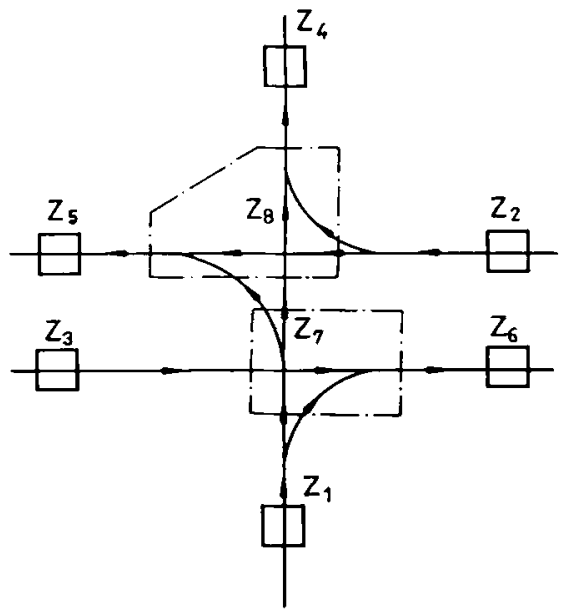

Fig. 25. The Inter-Merge structure.

\subsection{Remarks}

Several modulised floor-path nets for both uni- and bidirectional system have been established. The necessary properties for a robust AGVS have been embeded in these nets. Because the control-loop nets established in this paper are closed systems, the traffic control signals in the net are always there (strict conservation property), and, once the control function of the net is executed, the control tokens return to their initial places and wait for the next through vehicles (liveness properties). The flow-path nets reflect the real floor-path structure, and tokens in the nets reflect system vehicles. Hence, only at the moment when vehicles are in the net, can the net behaviour have these necessary properties. The number of vehicles from input zones to the net always results in the same number of vehicles from output zones out of the net. This ensures that the flow-path net keeps the conservation and liveness properties. Since each control-loop net is already a closed system, when several nets are connected together as a complete system, the connection is done among the flow-path nets only.

\section{Union Rules and Procedure}

The modulised floor-path nets developed above are satisfied with the system robustness properties - safeness, boundedness, strict conservation, reachability, and liveness within input and output zones. It is now necessary to see by the union of these nets, if a robust system model be built. Several union rules are described as follows:

Rule 1: Every output zone of a net has to be connected to the input zone of another net. 


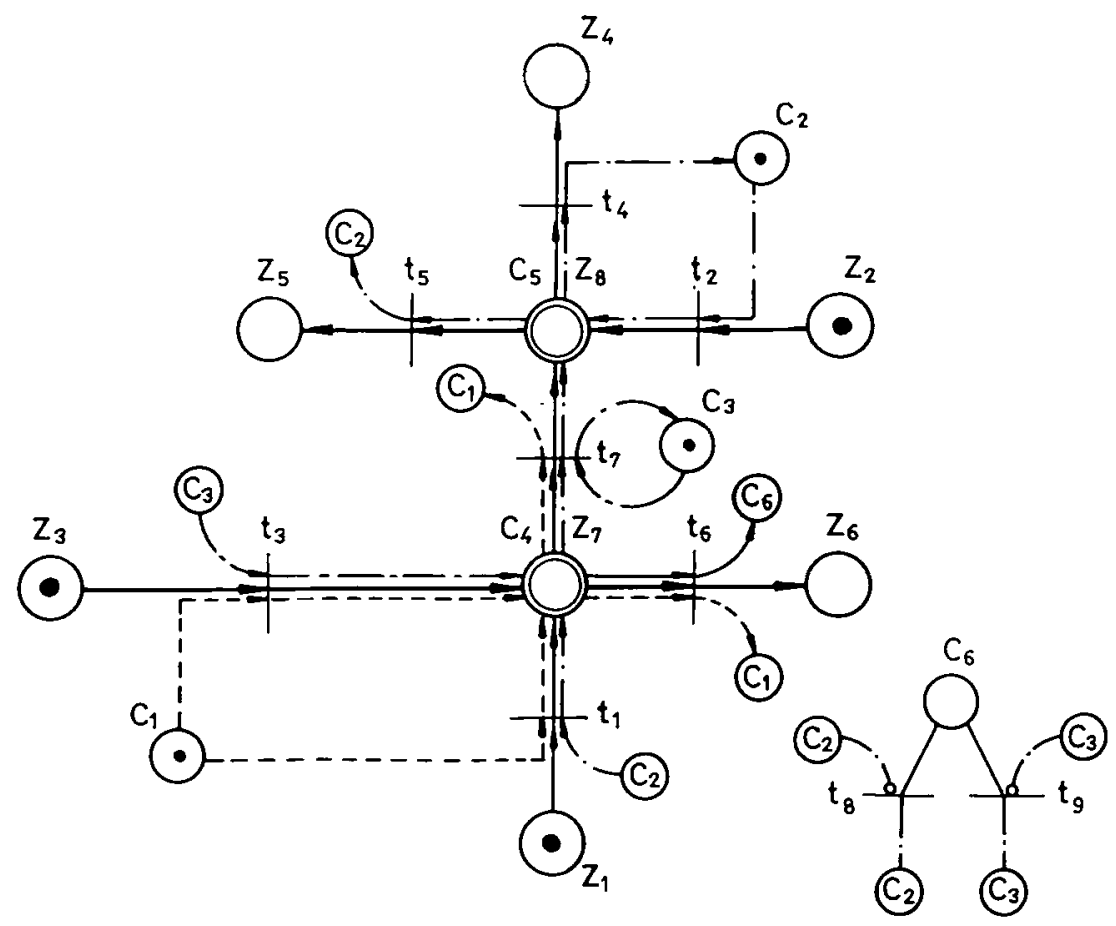

Fig. 26. The Inter-Merge net.

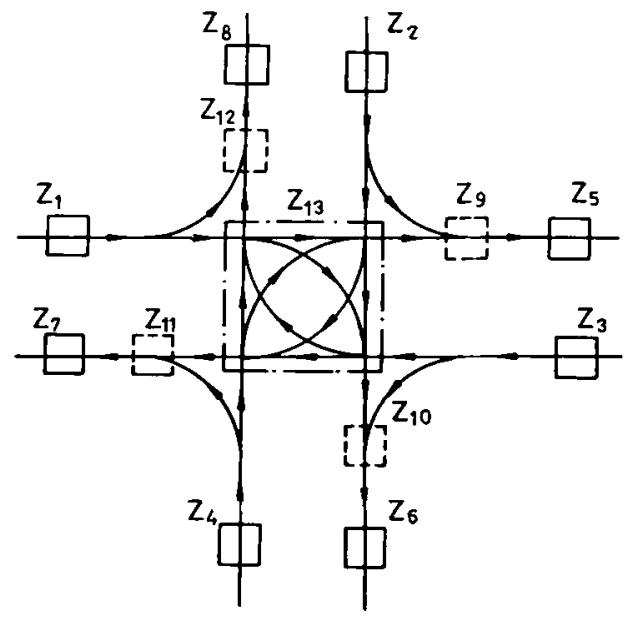

Fig. 27. The C-I-M structure.

Rule 2: Similarly, every input zone of a net has to be connected to the output zone of another net.

Rule 3: The output zone of a net cannot be connected with its input zone. Similarly, the input zone of a net cannot be connected with its output zone.

By following the three rules, all the necessary nets can be united as a complete closed-system model. This is because vehicles from other net output zones get into input zones of a net, and from output zones of the net get out of the net, and, again, get into another net input zones. This occurs repeatedly, and vehicles are always in the model. Therefore, the strict conservation property remains in the system after the union. According to the previous study, if a system is conservative, the system should be bounded. The zone control method is used to limit the vehicle number to 1 in a zone at any time. Hence, the safeness property is still there after the union. Since control-loop nets in the system are so independent, as long as every control-loop net can take care its traffic problems, the traffic problems of the whole system should be dealt with. Because each transition of the control-loop net is live and tokens (vehicles) in the flow-path nets keep moving, the deadlock problem never happens in the system; the complete model is live. If a system is live, the reachability property is, of course, there too. Thus, if an AGVS model is built by the union of the modulised floor-path nets developed in this paper, and the union rules are followed, the model will be robust at the foor level if the floor-path layout is robust.

The modelling procedure for a robust AGVS at floor level is described as follows:

1. Obtain a reasonable floor-path layout by any means.

2. Choose proper modulised floor-path nets according to the floor-path layout, and place the chosen nets at the proper location in the layout.

3. Connect the output (or input) zones of a net with the input (output) zones of the consecutive net.

4. Inspect whether the complete model after the union is a closed system or not, if not, go to step 2 or 3.

5. Do qualitative and quantitative analyses on the complete model by either analytical methods or simulation.

6. If the results indicate that the model is not robust, modify the floor-path layout and go to step 2 . 
(a)

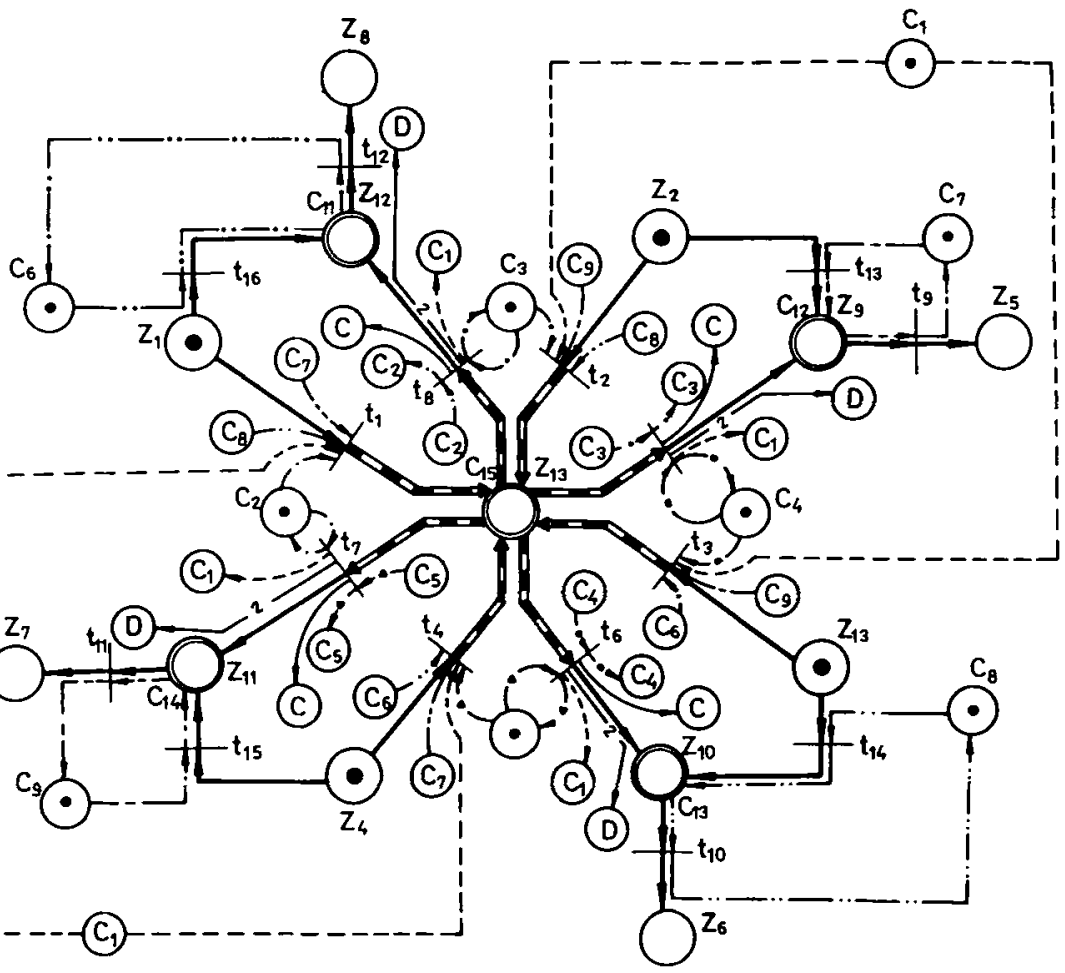

including 4 control lines and a physical line (b) $-----c_{1}$ control line

$-\cdot-\cdot-\mathrm{C}_{2}$ control line

-.... $\mathrm{C}_{3}$ control line

--- $\mathrm{C}_{4}$ control Iine

$\longrightarrow--\cdot-\mathrm{C}_{5}$ control line

$-\cdots-\cdots-\mathrm{C}_{6}$ control line

$--\longrightarrow C_{7}$ control line

$-\cdots-\cdots-\mathrm{C}_{8}$ control line

$-\cdots-C_{9}$ control line

$-2 \rightarrow$ (D) $\equiv=$ (D) refer to part (d)

(C) refer to part (c)

(c)

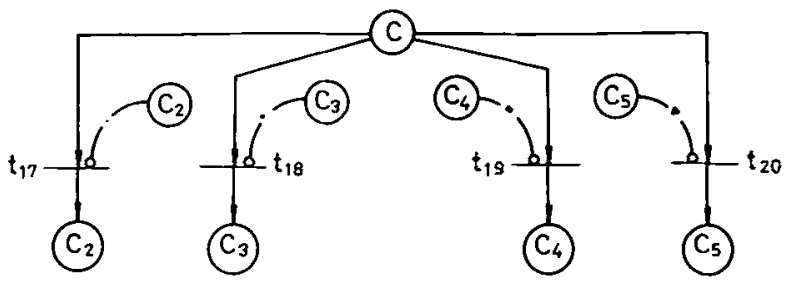

(d)

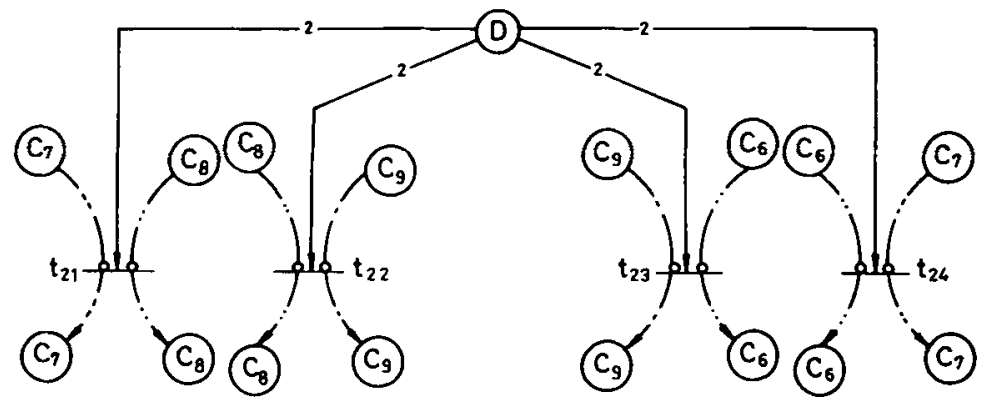

Fig. 28. (a) The C-I-M net. (b) The C-I-M auxiliary figure. (c) The C-I-M auxiliary C net. (d) The C-I-M auxiliary D net. 


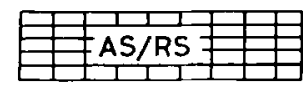

iil

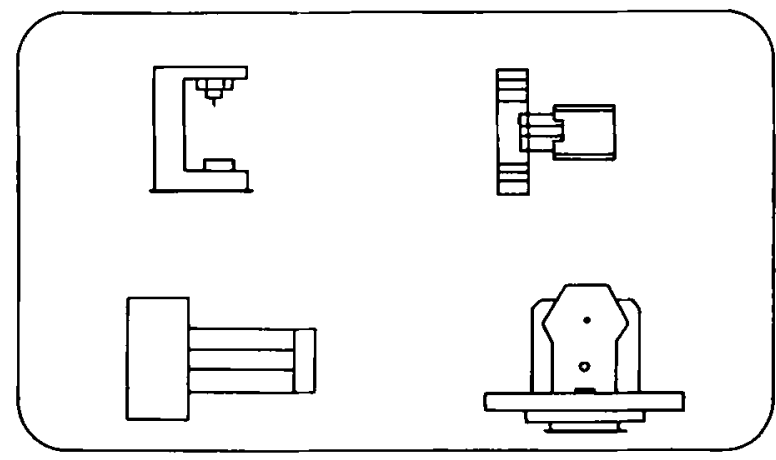

Fig. 29. The plant layout.

If the floor-path layout is robust, the AGVS model obtained by following steps 1 to 4 will be robust. If not, one can still use the above modelling procedure to develop an initial model. Based on the initial model, system analyses and simulations can be performed so that the model can be modified (step 5 to 7 ). By repeating steps 1 to 6 , very close to an ideal model can be obtained.

\section{Application}

Fig. 29 is a small manufacturing plant layout. The plant includes an automated storage/retrieval system and several $\mathrm{NC}$ or CNC workstations. The plant intends to adopt AGVS as their material handling system. After careful study, the AGVS floor-path layout is determined as shown in Fig. 30. The floor-path layout shows that there are $2 \mathrm{D}, 2 \mathrm{M}, 1 \mathrm{I}$ and several line structures in the system. Therefore, by following the union-procedure steps 1 to 4, the complete AGVS model is obtained (in Fig. 31). Then, analytical analyses or simulations can be carried out to verify the robustness of the net. Here, because the anlayses of the complete model may involve too much for this paper, half of the model is used to demonstrate how to verify the system robustness. A submodel including $1 \mathrm{D}, 1 \mathrm{M}$ and a line is chosen (see Fig. 31, cut along line $\mathrm{AA}$ and throw away the upper part), and transition $t_{11}$ and $t_{20}$, and $t_{14}$ and $t_{25}$ are overlapped, respectively, in order to keep the submodel closed (see Fig. 32). The safeness, boundedness, strict conservation and reachability properties will be examined in the following.

1. Safeness: Because of the modified firing rule that the output place cannot have a token in it, it is guaranteed that there will never be more than one big token (representing vehicles) in any place. Therefore, the safeness property is there.

2. Boundedness: if the model is conservative, the model is bounded.

3. Strict conservation: The incident matrix of Fig. 32 is

$$
\begin{aligned}
& \begin{array}{lllllllllll}
Z_{12} & Z_{13} & Z_{14} & Z_{15} & Z_{16} & Z_{17} & Z_{14} & Z_{19} & C_{3} & C_{6}
\end{array} \\
& N=\begin{array}{r}
t_{11} \\
t_{12} \\
t_{13} \\
t_{14} \\
t_{15} \\
t_{16} \\
t_{17} \\
t_{18} \\
t_{19}
\end{array}\left[\begin{array}{rrrrrrrrrr}
1 & 0 & 0 & 0 & 0 & 0 & -1 & 0 & 0 & 0 \\
-1 & 1 & 0 & 0 & 0 & 0 & 0 & 0 & 0 & 0 \\
0 & 0 & -1 & 0 & 0 & 1 & 0 & 0 & 0 & 0 \\
0 & -1 & 0 & 1 & 0 & 0 & 0 & 0 & 0 & 0 \\
0 & 0 & 0 & -1 & 1 & 0 & 0 & 0 & 0 & 0 \\
0 & 0 & 0 & 0 & 0 & -1 & 0 & 1 & -1 & 1 \\
0 & 0 & 0 & 0 & 0 & 0 & 1 & -1 & 1 & -1 \\
0 & 0 & 0 & 0 & -1 & 0 & 0 & 1 & -1 & 1
\end{array}\right]
\end{aligned}
$$

There exists a weighting vector $W=(1,1,1,1,1,1,1,1,1,1)^{\mathrm{T}}$ such that $N \cdot W=0$. Therefore, the model is strictly conservative and also bounded.

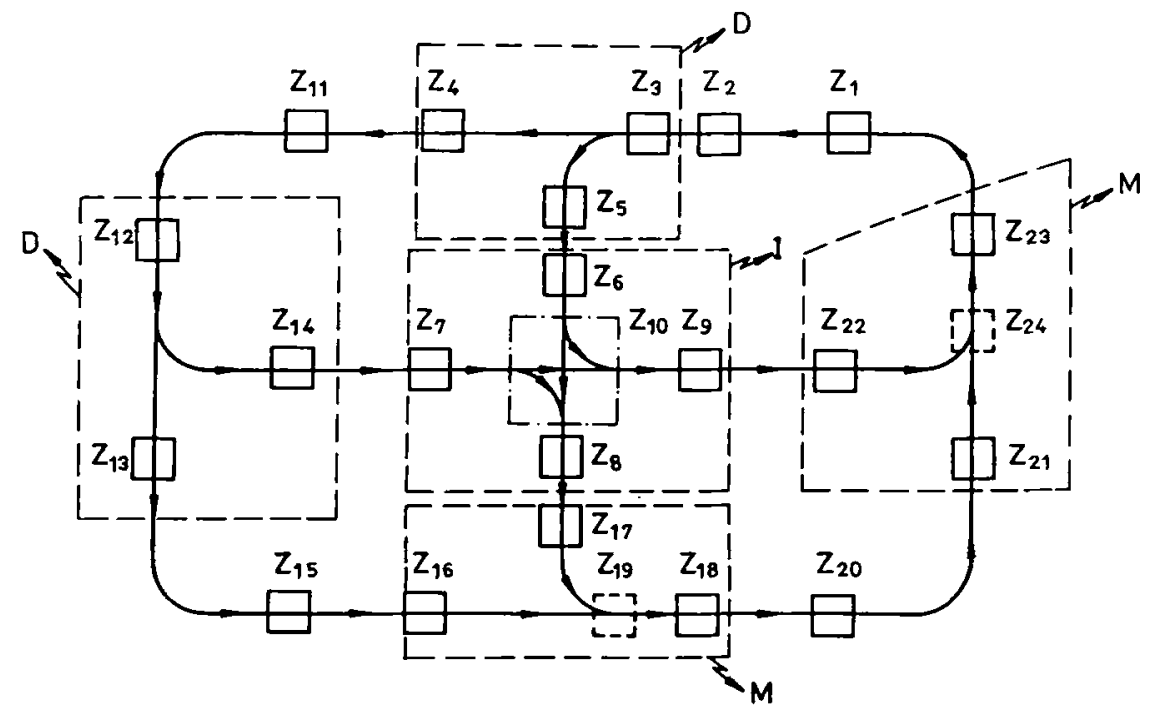

Fig. 30. The plant flow-path layout. 


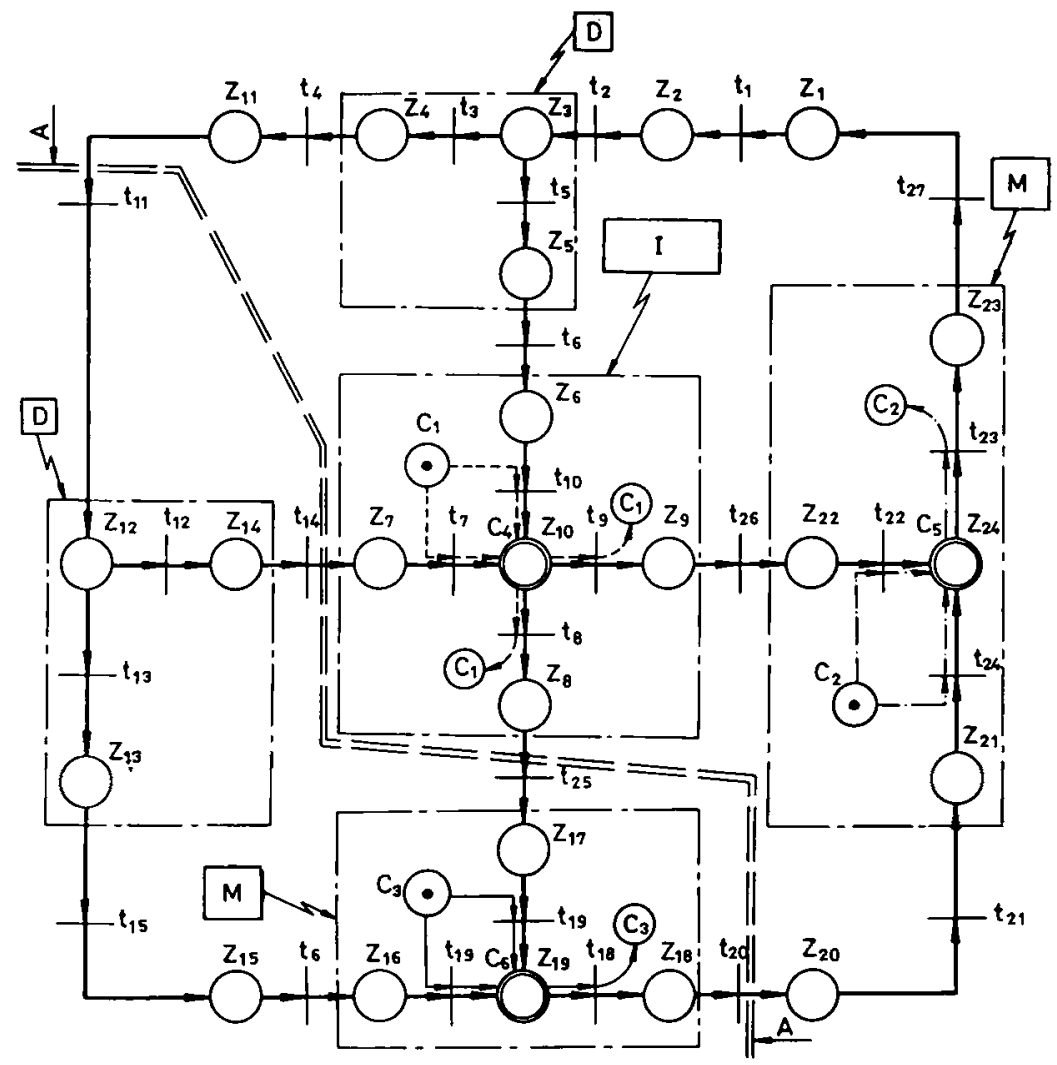

Fig. 31. The plant AGVS Petri-net model.

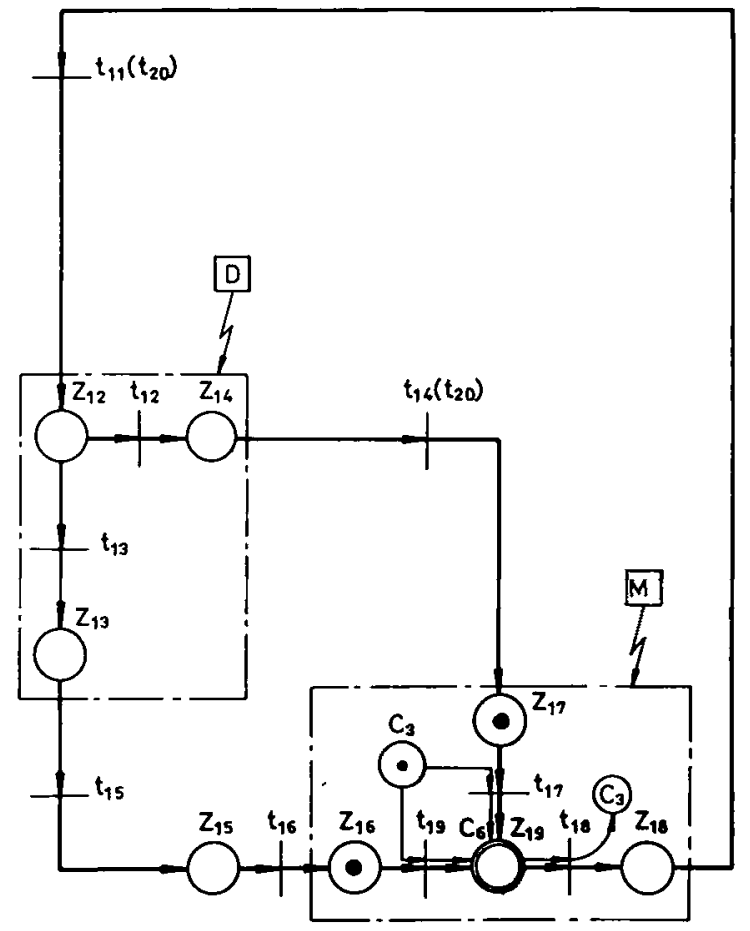


4. Liveness: In Fig. 32, there are two rotation cycles, $t_{17} t_{18} t_{11} t_{12} t_{14} t_{17}$ and $t_{19} t_{18} t_{11} t_{12} t_{13} t_{15} t_{16} t_{19}$, and all the transitions have been included in one or more rotation cycles. Therefore, the model is live.

5. Reachability: Since the model is live, all the paths are reachable.

The submodel obtained by the union of the divide, merge and line nets is proved to be robust. The complete model (Fig. 31) can be proved to be robust by a similar method. Intuitively, any model obtained by the union of the nets established in this paper should be robust.

\section{Conclusions}

An intelligent AGVS model tool has been developed in this paper. The AGVS robustness properties at floor level have been included in this tool. Therefore, the model developed by the tool will automatically be a robust model at floor level. More research regarding the robust AGVS model at other levels will be carried out in the future.

\section{References}

1. S. Hsieh and K.-H. M. Lin, "Building AGV Traffic-Control Models With Place-Transition Nets", The International Journal of Advanced Manufacturing Technology, 6, pp. 000-000, 1991.

2. S. Hsieh and K.-H. M. Lin, "AGVS Mechanism", to appear in the International Journal of Advanced Manufacturing Technology.

3. S. Hsieh and Y.-J. Shih, "AGVS and Its Petri-Net Properties", to appear in The Journal of Intelligent Manufacturing Systems.

4. J. L. Peterson, Petri Net Theory and the Modeling of Systems, Prentice-Hall, Englewood Cliffs, NJ 07632, 1981.

5. P. J. Egbelu and J. M. A. Tanchoco, "Potentials for Bi-directional Guide-Path for Automated Guided Vehicle Based Systems", International Journal of Production Research, 24(5), Pp. 1075-1097, 1986. 